\section{Desempenho dos municípios brasileiros quanto à oferta de serviços especializados em saúde bucal}

\author{
Performance of Brazilian municipalities in the \\ supply of specialized oral health services
}

\author{
Desempeño de los municipios brasileños \\ respecto a la oferta de servicios especializados \\ en salud bucal
}

Maria Helena Rodrigues Galvão 1

Angelo Giuseppe Roncalli 1

\section{Resumo}

Objetivou-se analisar em nível nacional os fatores associados à disponibilidade de serviços odontológicos especializados nos municípios brasileiros. É um estudo ecológico, com amostra de 776 municípios que participaram do 1o ciclo do Programa de Melhoria do Acesso e da Qualidade dos Centros de Especialidades Odontológicas (PMAQ-CEO) realizado em 2014. As variáveis dependentes do estudo consistiram em um coeficiente criado com base nas variáveis de número de profissionais e carga horária semanal de cirurgiões-dentistas atuando nas especialidades minimas por 10 mil habitantes. Realizou-se uma análise fatorial exploratória para a criação de um escore que mensura o desempenho dos municípios quanto à disponibilidade de serviços odontológicos especializados. A fim de avaliar os fatores associados ao desempenho dos municípios utilizou-se o teste do qui-quadrado de Pearson, tendo como variáveis independentes os seguintes indicadores municipais categorizados em tercis: renda per capita, Índice de Desenvolvimento Humano Municipal (IDH-M), população residente, despesa total com saúde por habitante e equipes de saúde bucal por 10 mil habitantes. O maior desempenho quanto à disponibilidade de serviços especializados em saúde bucal esteve associado a municípios com menor porte populacional (67,3\%; IC: 61,6-73,0; $p<0,001)$, com menor IDH-M (41,9\%; IC: 35, 8-48,0; $p<0,001)$ e com menor renda per capita (41,2\%; IC: 35,2-47,3; $p<0,001)$ e maior média de equipes de saúde bucal por 10 mil habitantes (50,6\%; IC: 46,0-58,4; $p<0,001)$. Os resultados demonstram impactos positivos da implantação da Politica Nacional de Saúde Bucal no país, como o cumprimento do objetivo de ampliação da oferta dos serviços de atenção secundária sob o princípio da equidade na assistência.

Serviços de Saúde Bucal; Qualidade, Acesso e Avaliação da Assistência à Saúde; Indicadores Compostos

\author{
Correspondência \\ M. H. R. Galvão \\ Universidade Federal do Rio Grande do Norte. \\ Av. Senador Salgado Filho 1787, Natal, RN 59056-000, Brasil. \\ mhrgalvao@gmail.com \\ 1 Universidade Federal do Rio Grande do Norte, Natal, Brasil.
}




\section{Introdução}

A expansão da atenção secundária em saúde bucal no Brasil representa uma das principais diretrizes da Política Nacional de Saúde Bucal, que teve como sua principal estratégia a criação dos Centros de Especialidades Odontológicas (CEO) 1. Esses Centros consistem em serviços de referência às equipes de saúde bucal na atenção básica para a resolução de casos complexos que demandam um atendimento especializado. O funcionamento desses CEO deve ser planejado de acordo com a realidade epidemiológica local e articulado com as equipes de saúde bucal da atenção básica 2 . Segundo informações do Cadastro Nacional de Estabelecimentos de Saúde, em janeiro de 2020 existiam 1.159 CEO implantados no país (Departamento de Informática do SUS. http://cnes2.datasus.gov.br/ Mod_Ind_Habilitacoes.asp?VEstado=00\&VTipo=H, acessado em 07/Jan/2020).

Entretanto, os desafios para a efetivação de uma atenção integral e resolutiva ultrapassam a implantação do serviço. A análise da disponibilidade de serviços é uma importante estratégia de avaliação das políticas públicas de saúde, sendo um indispensável componente para a avaliação do acesso aos serviços, com influência sobre a utilização e a acessibilidade. Nesse contexto, o Programa de Melhoria do Acesso e da Qualidade (PMAQ) desponta como um importante avanço no processo de institucionalização da avaliação no contexto do Sistema Único de Saúde (SUS). A criação do PMAQ-CEO teve por objetivo a melhoria no acesso e na qualidade nos CEO, além de criar uma base de dados pública com informações sobre os serviços em funcionamento no país 3.

Apesar disso, a maioria dos estudos que avaliaram os CEO utilizou o critério de realização de procedimentos por especialidade, por meio do indicador de Cumprimento Global de Metas, para mensurar o desempenho dos centros 4,5. Tais estudos mostram que há uma grande proporção de CEO com desempenho insatisfatório ou ruim quanto ao cumprimento das metas de produção ambulatorial propostas, tendo pior desempenho os municípios com menor população e menor Índice de Desenvolvimento Humano (IDH), evidenciando o baixo desempenho em populações de maior vulnerabilidade. Tal associação foi encontrada tanto em nível nacional 6 quanto em estudos no nível estadual ao avaliar os centros dos estados do Amazonas 7, Maranhão 8, Bahia 9 e Paraíba 10.

Torna-se necessária, portanto, a realização de estudos que avaliem outros indicadores de desempenho desses serviços que considerem, além da produção de procedimentos, a disponibilidade destes, tendo em conta as características populacionais dos municípios onde estão inseridos. Ressalta-se a inexistência na literatura de um escore específico que mensure o aspecto da disponibilidade de serviços odontológicos nos CEO.

O presente trabalho se propõe a avaliar o acesso aos CEO, com base na dimensão da disponibilidade, considerando o conceito de acesso definido por Penchansky \& Thomas 11 como o grau de ajuste que existe entre usuários e o sistema de saúde, podendo ser mensurado baseando-se em cinco dimensões: disponibilidade, acessibilidade, comodidade, capacidade aquisitiva e aceitabilidade, sendo a disponibilidade a relação entre quantidade e tipos de procedimentos e serviços oferecidos e quantidade e tipos de necessidades dos usuários.

Dessa forma, o objetivo deste trabalho foi analisar em nível nacional os fatores associados à disponibilidade de serviços odontológicos especializados nos municípios brasileiros.

\section{Método}

\section{Delineamento e contexto}

Trata-se de um estudo ecológico, em nível de agregação municipal, com a análise de dados secundários provenientes da fase de avaliação externa do primeiro ciclo do PMAQ-CEO. Coletaram-se dados referentes a $932 \mathrm{CEO}$, localizados em 776 municípios, consistindo em um censo dos serviços implantados no país. O período de coleta de dados foi relativo ao primeiro semestre de 2014. 


\section{Fonte de dados}

Utilizou-se o banco de dados referente ao módulo VII do Instrumento de Avaliação Externa para os Centros de Especialidades Odontológicas. O banco de dados é público e está disponível na página de Internet da Secretaria de Atenção Primária à Saúde, Ministério da Saúde (http://aps.saude.gov. $\mathrm{br} / \mathrm{ape} / \mathrm{pmaq} / \mathrm{ciclo1ceo/,} \mathrm{acessado} \mathrm{em} \mathrm{02/Fev/2018).} \mathrm{As} \mathrm{variáveis} \mathrm{dependentes} \mathrm{selecionadas} \mathrm{foram}$ o número de profissionais por especialidade e a carga horária semanal dos cirurgiões-dentistas por especialidade. Consideraram-se para análise as especialidades mínimas preconizadas pela Portaria de Consolidação no 5, de 28 de setembro de 2017 3: cirurgia oral, periodontia, endodontia, atendimento a pacientes com necessidades especiais e estomatologia.

\section{Criação do escore de desempenho dos municípios}

Para análise do banco de dados, previamente foi realizada análise univariada de valores atípicos por meio de escores padrão, seguida da multivariada usando-se a medida D2 de Mahalanobis, sendo excluídos os dados atípicos considerados como erro de procedimento. Após a análise da perda de dados, optou-se pela exclusão de um CEO por não apresentar dados para as variáveis de interesse, e das variáveis relacionadas à especialidade "Estomatologia”, por estarem ausentes em 34,4\% dos CEO, comprometendo a aplicação de técnicas multivariadas de análise. As demais variáveis apresentaram valores de perdas abaixo de $1 \%$ e, constatada a aleatoriedade destas perdas, foram considerados como ignoráveis (Secretaria de Atenção Primária à Saúde, Ministério da Saúde. http://aps.saude.gov.br/ ape/pmaq/ciclo1ceo/, acessado em 02/Fev/2018).

Procedeu-se à criação de um coeficiente que expressasse a quantidade de profissionais por especialidade por 10 mil habitantes, bem como a carga horária disponibilizada para o atendimento por especialidade por 10 mil habitantes. Para tanto, o número de profissionais e a carga horária de atendimento por especialidade foram somados por município, divididos pela população estimada do município no ano de 2014 e multiplicados por 10 mil. A escolha da constante 10 mil na composição do coeficiente se deu devido à melhor adequação para a apresentação dos resultados, evitando que o resultado seja expresso em um número decimal de difícil compreensão ${ }^{12}$. Posteriormente, realizou-se uma análise fatorial exploratória, utilizando como variáveis indicadoras os coeficientes previamente criados, com a finalidade de obter a redução de dados por meio da observação das estruturas latentes com a formação de fatores que representam o conjunto original de variáveis. Para isso, optou-se por fazer uma análise fatorial realizando inicialmente uma matriz de correlação entre as variáveis de interesse. Todas as variáveis apresentaram valores de correlação maiores que 0,30 e foram incluídas no modelo de análise. A variância total explicada pelo fator extraído pelo modelo final foi de 72,6\%.

Com a finalidade de verificar a validade do modelo obtido utilizou-se a técnica descrita por Hair et al. 13. Realizou-se uma análise fatorial exploratória com duas subamostras aleatórias correspondentes a aproximadamente $50 \%$ dos casos cada, contendo a subamostra 1 (364 municípios) e a subamostra 2 (395 municípios). Observou-se nas subamostras valores de correlação maiores que 0,30. As comunalidades e as cargas fatoriais observadas foram semelhantes ao modelo inicial e um único fator foi extraído em ambos os casos. A variância total explicada pelo fator extraído por cada modelo obtido por meio das subamostras foi de $72,1 \%$ para a subamostra 1 e de $72,9 \%$ para a subamostra 2 . Durante a análise fatorial optou-se pelo método listwise para a exclusão de casos com dados ausentes para uma das variáveis incluídas no modelo, totalizando ao final da análise 759 municípios. Posteriormente, utilizaram-se os escores fatoriais obtidos na análise para a composição de um escore de disponibilidade de serviços odontológicos especializados nos municípios por meio da fórmula a seguir:

$$
\text { Escore de disponibilidade: }\left(\frac{x-\bar{x}}{s}\right) \cdot 10+50
$$

O objetivo desse ajuste no escore foi criar uma variável com valores sempre positivos, pois a variável gerada pela análise fatorial é adimensional e inclui valores negativos e positivos. Desse modo, cada valor da variável gerada (fator) é subtraído de sua média e dividido pelo seu desvio padrão gerando um valor normalizado e, em seguida, multiplicado por 10 e somado a 50. Isso produz uma variável sempre positiva, com média igual a 50 e desvio padrão igual a 10. Após essa fase, os municípios foram 
classificados quanto a esse escore de desempenho, utilizando como pontos de corte os quartis, sendo esta a variável dependente do estudo. Os quartis obedecem a uma escala crescente de desempenho, de modo que o quartil inferior se refere ao pior desempenho e o superior ao melhor desempenho.

As variáveis independentes foram agrupadas em duas dimensões: (1) características sociodemográficas dos municípios e (2) organização dos serviços de saúde dos municípios. Na primeira dimensão, incluíram-se as variáveis "população estimada", "Índice de Desenvolvimento Humano Municipal (IDH-M)" e "renda per capita". Na segunda, foram incluídas as variáveis "equipes de saúde bucal por 10 mil habitantes" e "despesa total com saúde por habitante". A fonte dos dados para as variáveis IDH-M e renda per capita foi o Atlas do Desenvolvimento Humano no Brasil 14, tendo como referência o ano de 2010. Para as variáveis "população estimada", "equipes de saúde bucal por 10 mil habitantes", "despesa total com saúde por habitante" e "número de cadeiras de CEO", utilizou-se como referência o ano de 2014, tendo por fonte de dados o Departamento de Informática do SUS (DATASUS. http://www2. datasus.gov.br/DATASUS/index.php?area=0202, acessado em 02/Fev/2018).

\section{Análise dos dados}

Realizou-se uma análise descritiva das variáveis independentes e, posteriormente, procedeu-se à categorização destas, utilizando como ponto de corte os tercis. Para a avaliação da relação entre a variável dependente e as independentes, realizou-se uma análise bivariada por meio do teste qui-quadrado de Pearson, com nível de significância igual a 5\%. Para a análise de dados foi usado o software Stata versão 14.0 (https://www.stata.com/).

\section{Resultados}

Com relação à avaliação da disponibilidade de serviços de atenção secundária, analisaram-se 776 municípios que contam com CEO. A população nesses municípios variou de 4.451 a 11.376 .685 habitantes, 50\% dos municípios tinham até 49.510 habitantes. O IDHM médio dos municípios foi de 0,693, e a renda per capita média de $\mathrm{R} \$ 612,80$. Quanto à oferta de serviços de saúde bucal, observou-se uma média de 1,9 equipe de saúde bucal e 1,1 cadeira de CEO para cada 10 mil habitantes. A despesa total de saúde por habitante nos municípios variou entre R\$ 195,50 e R \$ 3.710,50, em que 50\% dos municípios avaliados gastaram até $\mathrm{R} \$ 531,70$ por habitante, conforme Tabela 1.

No que se refere à disponibilidade de atendimento por especialidade, constatou-se que a Endodontia tem a maior média de profissionais, com 0,5 dentista e carga horária semanal de 10,4 horas por 10 mil habitantes. A Estomatologia é a especialidade com a menor disponibilidade, com 0,2 profissional e carga horária semanal de 2,9 horas para cada 10 mil habitantes (Tabela 2).

Obtiveram-se valores de correlação (r) com valores acima de 0,300 entre as variáveis de interesse do estudo. $O$ teste de esfericidade de Bartlett foi significativo $(\mathrm{p}>0,001)$ e o valor da estatística Kaiser-Meyer-Olkin $(\mathrm{KMO}=0,8199)$ indicou a adequação da amostra como "muito boa" para a análise fatorial 13 .

A Tabela 3 apresenta as cargas fatorais e os valores da comunalidade para as variáveis do estudo, para os 759 municípios incluídos na análise fatorial. A análise resultou na extração de um fator que representa a redução de dimensão das variáveis incluídas no modelo. Observou-se uma carga fatorial acima de 0,80 e comunalidade acima de 0,50 para todas as variáveis inseridas no modelo final para a representação do fator obtido.

Após a avaliação dos fatores associados ao desempenho dos municípios quanto à disponibilidade de serviços odontológicos especializados, identificou-se que aqueles com menor porte populacional apresentaram maior proporção de municípios com maior desempenho (Q4 = 67,3\%; IC: 61,6-73,0; $\mathrm{p}<0,001)$, já os com maior porte populacional obtiveram maior proporção de municípios com o menor desempenho (Q1 = 69,6\%; IC: 63,9-75,4; p < 0,001). Com relação ao IDH-M, aqueles com menor IDH apresentaram maior proporção de municípios com maior desempenho (Q4 = 41,9\%; IC 35,8-48,0; $\mathrm{p}<0,001)$ e os com maior IDH-M obtiveram, em sua maioria, o menor desempenho $(43,3 \% ;$ IC: $37,2-49,4)$. No tocante à renda, municípios com menor renda per capita apresentaram em 
Tabela 1

Características sociodemográficas e dos serviços de saúde dos municípios onde estão localizados os Centros de Especialidades Odontológicas (CEO). Brasil, 2010.

\begin{tabular}{|c|c|c|c|c|c|}
\hline $\begin{array}{l}\text { Características sociodemográficas e dos serviços de saúde dos } \\
\text { municípios }(n=776)\end{array}$ & Mínimo & Máximo & Média & DP & Mediana \\
\hline População estimada do município & 4.451 & 11.376 .685 & 149.420 & $531.763,67$ & 49.510 \\
\hline IDH-M & 0,520 & 0,860 & 0,693 & 0,741 & 0,708 \\
\hline Renda per capita ( $\mathrm{R} \$)$ & 176,8 & $2.043,7$ & 612,8 & 309,9 & 606,7 \\
\hline Equipes de saúde bucal por 10 mil habitantes & 0,0 & 6,8 & 1,9 & 1,4 & 1,8 \\
\hline Despesa total em saúde por habitante (R\$) & 195,5 & $3.710,5$ & 599,4 & 269,3 & 531,7 \\
\hline Número de cadeiras de CEO por 10 mil habitantes (2014) & 0,06 & 6,61 & 1,09 & 0,91 & 0,85 \\
\hline
\end{tabular}

DP: desvio padrão; IDH-M: Índice de Desenvolvimento Humano Municipal.

\section{Tabela 2}

Características da disponibilidade de serviços nos municípios por especialidades dos Centros de Especialidades Odontológicas (CEO), segundo avaliação externa do Programa de Melhoria do Acesso e da Qualidade dos Centros de Especialidades Odontológicas (PMAQ-CEO). Brasil, 2014.

\begin{tabular}{|c|c|c|c|c|c|}
\hline & Mínimo & Máximo & Média & DP & Mediana \\
\hline \multicolumn{6}{|l|}{ Número de profissionais por 10 mil habitantes } \\
\hline Cirurgia oral & 0,00 & 2,65 & 0,37 & 0,33 & 0,27 \\
\hline Endodontia & 0,00 & 3,41 & 0,50 & 0,47 & 0,38 \\
\hline Estomatologia & 0,00 & 2,58 & 0,19 & 0,28 & 0,08 \\
\hline Pacientes com necessidades especiais & 0,00 & 3,99 & 0,33 & 0,39 & 0,22 \\
\hline Periodontia & 0,00 & 2,65 & 0,31 & 0,31 & 0,23 \\
\hline \multicolumn{6}{|c|}{ Carga horária semanal dos profissionais por 10 mil habitantes } \\
\hline Cirurgia oral & 0,00 & 52,92 & 7,18 & 7,58 & 4,69 \\
\hline Endodontia & 0,00 & 72,07 & 10,04 & 10,05 & 7,14 \\
\hline Estomatologia & 0,00 & 45,26 & 2,94 & 4,97 & 1,08 \\
\hline Pacientes com necessidades especiais & 0,00 & 54,67 & 6,31 & 7,51 & 3,84 \\
\hline Periodontia & 0,00 & 52,92 & 6,16 & 6,58 & 4,20 \\
\hline
\end{tabular}

DP: desvio padrão.

\section{Tabela 3}

Cargas fatoriais obtidas pela análise fatorial exploratória para as variáveis de disponibilidade dos serviços especializados em saúde bucal nos municípios com Centros de Especialidades Odontológicas (CEO) implantados. Brasil, 2014.

Cargas fatoriais Comunalidades

Profissionais de cirurgia oral por 10 mil habitantes

Profissionais de endodontia por 10 mil habitantes

Profissionais de pacientes com necessidades especiais por 10 mil habitantes

Profissionais de periodontia por 10 mil habitantes

Carga horária semanal dos profissionais de cirurgia oral por 10 mil habitantes

Carga horária semanal dos profissionais de endodontia por 10 mil habitantes

Carga horária semanal dos profissionais de pacientes com necessidades especiais por 10 mil habitantes

Carga horária semanal dos profissionais de periodontia por 10 mil habitantes

$\begin{array}{ll}0,879 & 0,773 \\ 0,839 & 0,703 \\ 0,810 & 0,657 \\ 0,856 & 0,733 \\ 0,877 & 0,770 \\ 0,858 & 0,737 \\ 0,832 & 0,693 \\ 0,862 & 0,742\end{array}$


sua maior parte melhor desempenho (Q4 = 41,2\%; IC: 35,2-47,3; $\mathrm{p}<0,001$ ) e os com maior renda per capita um menor desempenho (Q1 = 40,2\%; IC: 34,1-46,2; p < 0,001) (Tabela 4 e Figura 1).

Quanto à organização do sistema de saúde, verificou-se que municípios com maior número de equipes de saúde bucal por habitantes obtiveram melhor desempenho (Q4 = 50,6\%; IC: 46,0-58,4; p < $0,001)$ quanto à disponibilidade de serviços de atenção secundária em saúde bucal. Os municípios com menor quantidade de equipes de saúde bucal apresentaram maior proporção do menor desempenho (Q1 = 51\%; IC: 44,8-57,2; p < 0,001). Com relação à despesa total com saúde por habitante, não se observou um efeito dose-resposta, não estando clara a relação entre o grau de investimento em saúde e o desempenho dos CEO (Tabela 4 e Figura 1).

Tabela 4

Fatores associados ao desempenho dos municípios quanto à disponibilidade de serviços dos Centros de Especialidades Odontológicas (CEO). Brasil, 2014.

\begin{tabular}{|c|c|c|c|c|c|c|}
\hline & $\begin{array}{c}\text { Q1 } \\
(n=189) \\
\%(I C)\end{array}$ & $\begin{array}{c}\text { Q2 } \\
(n=191) \\
\%(I C)\end{array}$ & $\begin{array}{c}\text { Q3 } \\
(n=190) \\
\%(I C)\end{array}$ & $\begin{array}{c}\text { Q4 } \\
(n=189) \\
\%(I C)\end{array}$ & Total & Valor de $p$ \\
\hline População estimada do município (habitantes) & & & & & & $<0,001$ \\
\hline 82.719 e mais & $\begin{array}{c}69,6 \\
(63,9-75,4)\end{array}$ & $\begin{array}{c}27,1 \\
(21,6-32,7)\end{array}$ & $\begin{array}{c}2,4 \\
(0,5-4,3)\end{array}$ & $\begin{array}{c}0,8 \\
(0,0-1,9)\end{array}$ & 247 & \\
\hline De 32.271 a 82.718 & $\begin{array}{c}5,9 \\
(3,0-8,8)\end{array}$ & $\begin{array}{c}43,1 \\
(37,1-49,2)\end{array}$ & $\begin{array}{c}45,5 \\
(39,4-51,6)\end{array}$ & $\begin{array}{c}5,5 \\
(2,7-8,3)\end{array}$ & 255 & \\
\hline Até 32.270 & $\begin{array}{c}0,8 \\
(0,0-1,9)\end{array}$ & $\begin{array}{c}5,4 \\
(2,7-8,2)\end{array}$ & $\begin{array}{c}26,5 \\
(21,1-31,9)\end{array}$ & $\begin{array}{c}67,3 \\
(61,6-73,0)\end{array}$ & 257 & \\
\hline IDH-M & & & & & & $<0,001$ \\
\hline 0,740 e mais & $\begin{array}{c}43,3 \\
(37,2-49,4)\end{array}$ & $\begin{array}{c}31,5 \\
(25,8-37,2)\end{array}$ & $\begin{array}{c}16,1 \\
(11,6-20,7)\end{array}$ & $\begin{array}{c}9,1 \\
(5,5-12,6)\end{array}$ & 254 & \\
\hline De 0,653 a 0,739 & $\begin{array}{c}24,6 \\
(19,3-29,9)\end{array}$ & $\begin{array}{c}28,2 \\
(22,6-33,7)\end{array}$ & $\begin{array}{c}23,4 \\
(18,2-28,6)\end{array}$ & $\begin{array}{c}23,8 \\
(18,6-29,1)\end{array}$ & 252 & \\
\hline Até 0,652 & $\begin{array}{c}6,7 \\
(3,6-9,8)\end{array}$ & $\begin{array}{c}15,8 \\
(11,3-20,3)\end{array}$ & $\begin{array}{c}35,6 \\
(29,7-41,5)\end{array}$ & $\begin{array}{c}41,9 \\
(35,8-48,0)\end{array}$ & 253 & \\
\hline Renda per capita $(\mathrm{R} \$)$ & & & & & & $<0,001$ \\
\hline 741,65 & $\begin{array}{c}40,2 \\
(34,1-46,2)\end{array}$ & $\begin{array}{c}30,1 \\
(24,4-35,8)\end{array}$ & $\begin{array}{c}17,7 \\
(12,9-22,4)\end{array}$ & $\begin{array}{c}12,0 \\
(8,0-16,1)\end{array}$ & 249 & \\
\hline De 392,06 a 741,64 & $\begin{array}{c}28,1 \\
(22,5-33,6)\end{array}$ & $\begin{array}{c}27,7 \\
(22,2-33,5)\end{array}$ & $\begin{array}{c}23,3 \\
(12,7-22,1)\end{array}$ & $\begin{array}{c}20,9 \\
(7,9-15,8)\end{array}$ & 253 & \\
\hline Até 392,05 & $\begin{array}{c}7,0 \\
(3,9-10,1)\end{array}$ & $\begin{array}{c}17,9 \\
(13,2-22,6)\end{array}$ & $\begin{array}{c}33,9 \\
(28,1-39,6)\end{array}$ & $\begin{array}{c}41,2 \\
(35,2-47,3)\end{array}$ & 257 & \\
\hline \multicolumn{7}{|l|}{ Organização do sistema de saúde } \\
\hline Equipes de saúde bucal por 10 mil habitantes & & & & & & $<0,001$ \\
\hline 2,47 e mais & $\begin{array}{c}2,7 \\
(0,8-4,9)\end{array}$ & $\begin{array}{c}14,8 \\
(10,8-19,7)\end{array}$ & $\begin{array}{c}31,9 \\
(27,1-38,8)\end{array}$ & $\begin{array}{c}50,6 \\
(46,0-58,4)\end{array}$ & 257 & \\
\hline De 1,10 a 2,46 & $\begin{array}{c}22,0 \\
(16,9-27,0)\end{array}$ & $\begin{array}{c}32,2 \\
(26,4-37,9)\end{array}$ & $\begin{array}{c}29,8 \\
(24,2-35,4)\end{array}$ & $\begin{array}{c}16,1 \\
(11,6-20,6)\end{array}$ & 255 & \\
\hline Até 1,09 & $\begin{array}{c}51,0 \\
(44,8-57,2)\end{array}$ & $\begin{array}{c}28,7 \\
(23,1-34,4)\end{array}$ & $\begin{array}{c}13,0 \\
(8,8-17,1)\end{array}$ & $\begin{array}{c}7,3 \\
(4,0-10,5)\end{array}$ & 247 & \\
\hline Despesa total em saúde por habitante $(R \$)$ * & & & & & & 0,001 \\
\hline 627,34 e mais & $\begin{array}{c}30,6 \\
(24,9-36,2)\end{array}$ & $\begin{array}{c}25,4 \\
(20,0-30,8)\end{array}$ & $\begin{array}{c}18,3 \\
(13,5-23,0)\end{array}$ & $\begin{array}{c}25,8 \\
(20,4-31,2)\end{array}$ & 252 & \\
\hline De 459,65 a 627,33 & $\begin{array}{c}19,2 \\
(14,3-24,1)\end{array}$ & $\begin{array}{c}21,6 \\
(16,5-26,7)\end{array}$ & $\begin{array}{c}29,2 \\
(23,6-34,8)\end{array}$ & $\begin{array}{c}30,0 \\
(24,3-35,7)\end{array}$ & 250 & \\
\hline Até 459,64 & $\begin{array}{c}24,6 \\
(23,7-34,9)\end{array}$ & $\begin{array}{c}28,5 \\
(23,0-34,0)\end{array}$ & $\begin{array}{c}27,7 \\
(22,3-33,2)\end{array}$ & $\begin{array}{c}19,1 \\
(14,3-24,0)\end{array}$ & 256 & \\
\hline
\end{tabular}

IC: intervalo de confiança; IDH-M: Índice de Desenvolvimento Humano Municipal.

Nota: teste de qui-quadrado de Pearson.

* Um dos municípios não apresentou os valores gastos com despesa em saúde no período analisado. 
Figura 1

Distribuição dos quartis de desempenho dos Centros de Especialidades Odontológicas (CEO) quanto à disponibilidade de serviço, de acordo com as características socioeconômicas e de serviços de saúde dos municípios. Brasil, 2014.

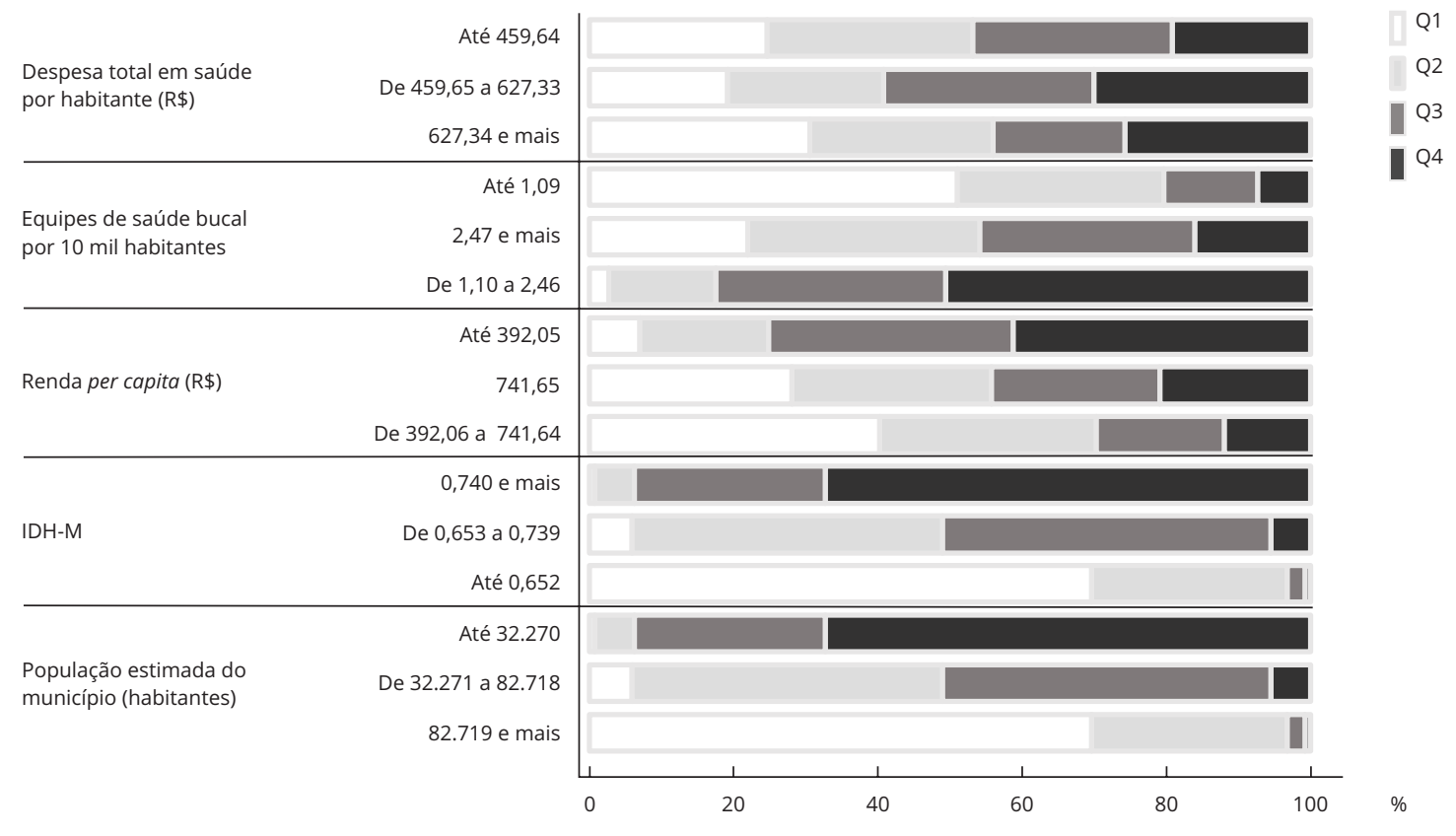

IDH-M: Índice de Desenvolvimento Humano Municipal.

\section{Discussão}

Os resultados do presente trabalho apontam que o melhor desempenho quanto à disponibilidade de atenção secundária em saúde bucal esteve associado a municípios com os piores indicadores socioeconômicos e municípios com uma melhor estruturação da rede de atenção em saúde bucal. Isso em certa medida demonstra o efeito indutor da Política Nacional de Saúde Bucal ${ }^{1}$ na ampliação e qualificação da atenção secundária, especialmente em municípios vulneráveis.

Todas as especialidades analisadas contribuíram significativamente na composição da variável final, o que significa que a carga horária e o número de profissionais para as especialidades de endodontia, cirurgia oral, periodontia e pacientes com necessidades especiais explicam a variância do desempenho dos municípios com relação à disponibilidade de serviços odontológicos especializados.

Os municípios com as maiores taxas de equipes de saúde bucal por 10 mil habitantes obtiveram um maior desempenho quanto à oferta de serviços especializados nos CEO. No Brasil, o aumento de indicadores de utilização de serviços odontológicos nos municípios esteve associado ao aumento das taxas de equipes de saúde bucal na Estratégia Saúde da Família 15. Além disso, uma maior cobertura de atenção primária no território onde os centros estão implantados consiste em fator relevante para a integralidade na assistência à saúde bucal, aumentando as chances de continuidade do tratamento odontológico no nível especializado 16.

Constatou-se uma baixa disponibilidade de serviços especializados ao avaliar a média de carga horária e o número de profissionais atuando nas especialidades por 10 mil habitantes nos municípios avaliados. Não existe uma normativa sobre a relação ideal entre profissional dos CEO por habitante no país, tendo em vista que a relação entre o número de profissionais por habitante deve considerar o contexto epidemiológico e socioeconômico local. A Portaria de Consolidação no 5 3, que apresenta 
critérios e parâmetros para o planejamento e programação de ações e serviços de saúde no âmbito do SUS, baseia-se no número de procedimentos especializados em saúde bucal por habitante, bem como na cobertura percentual de tais procedimentos como critério que norteia os processos de planejamento, programação, monitoramento e avaliação. Entretanto, o número de dentistas no SUS é apontado como o fator crítico no processo de trabalho odontológico. De acordo com Celeste et al. 17, municípios com a menor relação dentista por habitante apresentaram 3,23 vezes mais chances de não realizar procedimentos odontológicos básicos.

Os resultados encontrados neste trabalho divergem de alguns estudos publicados sobre avaliação dos CEO. Os fatores encontrados nos estudos associados ao desempenho dos CEO foram IDH-M, porte populacional, macrorregião geográfica e tempo de implantação do serviço. As localidades mais desenvolvidas socialmente e com maior porte populacional têm um melhor resultado na avaliação do desempenho dos CEO 4,5. Entretanto, o desempenho dos CEO mensurado em tais estudos está relacionado ao cumprimento de metas por procedimentos especializados, e não à capacidade de oferta de procedimentos.

Embora a utilização de serviços seja uma expressão da disponibilidade destes, esta relação não é diretamente observada, visto que a utilização, especialmente, dos serviços especializados pressupõe a necessidade de uso, além de sofrer influência de outros fatores organizacionais da rede de atenção, como os processos de referência e contrarreferência, organização da demanda para atendimento e regulação. Isso explica o fato de municípios com maior porte populacional obterem melhor desempenho nos estudos que utilizam o volume de procedimentos realizados, e na avaliação da disponibilidade de profissionais e carga horária semanal por habitante atingirem os piores resultados.

A avaliação do desempenho por meio da disponibilidade de serviços deve ser realizada de forma complementar à avaliação da produção de procedimentos e cobertura populacional, proposta na Portaria de Consolidação no 53 , por expressar a capacidade do serviço de ofertar atendimento, por intermédio da disponibilidade de profissionais, à população com base nas necessidades por elas apresentadas, sem penalizar os municípios de menor porte populacional.

O fortalecimento das redes de atenção à saúde bucal e a formação de consórcios intermunicipais podem superar a lacuna entre disponibilidade e utilização nos municípios de menor porte populacional, e a criação de novos serviços e o aumento de profissionais em municípios de maior porte podem melhorar a disponibilidade de atenção secundária, reduzindo as filas de espera para atendimento.

A integralidade pode ser definida como a garantia da continuidade da atenção nos distintos níveis de complexidade. A efetivação desse conceito pressupõe a disponibilidade de um profissional capaz de suprir as necessidades de saúde da população em todos os níveis de atenção. O presente estudo demonstra que existe uma diferença na disponibilidade de serviços odontológicos especializados em função das características sociodemográficas e dos serviços de saúde dos municípios, o que pode comprometer o princípio da integralidade na Rede de Atenção em Saúde Bucal. Além disso, é importante ressaltar que os resultados desta pesquisa se aplicam aos municípios que têm ao menos um CEO implantado, tendo em consideração que a maior parte dos municípios brasileiros não conta com tal serviço, que tende a ser concentrado em capitais e municípios de referência das regiões de saúde 18.

Além disso, a avaliação de produtividade proposta pelo Ministério da Saúde, por meio da Portaria de Consolidação no 5 3, carece de revisão e complementação ao considerar o tempo de publicação e os resultados obtidos. É importante que seja proposto um modelo avaliativo que contemple as especificidades e o potencial que cada serviço tem em cumprir tais metas, levando em consideração o porte populacional e as necessidades de saúde da população, bem como a aplicabilidade em diferentes contextos 19 .

A potencialidade deste estudo é o caráter abrangente, sendo incluídos todos os municípios com CEO em funcionamento no país. Ressaltam-se o rigor metodológico e a utilização de instrumentos validados e comparáveis, o que confere confiabilidade aos dados e a importância do estudo na criação de um indicador agregado de avaliação da disponibilidade de atenção secundária nos CEO do país, subsidiando a criação de novos indicadores de disponibilidade de serviços de atenção à saúde. $\mathrm{O}$ percurso metodológico adotado neste trabalho tem o potencial de ser aplicado na criação de outros indicadores de disponibilidade de serviços de saúde, usando coeficientes padronizados por número de habitantes, a utilização da análise fatorial e a transformação das cargas fatoriais em um escore, com possibilidade de aplicação em outras áreas. 
As potenciais fontes de viés de seleção dos participantes da amostra foram minimizadas devido ao alto número de serviços que aderiram à proposta do PMAQ-CEO, consistindo em um censo de todos os municípios com CEO em atividade no país no momento da coleta de dados. O caráter avaliativo da fase de coleta de dados do PMAQ-CEO pode ser apontado como uma potencial fonte de viés de informação, entretanto, espera-se que o efeito deste tenha ocorrido de forma aleatória.

Por fim, observou-se por meio deste estudo que uma maior oferta de serviços odontológicos especializados esteve associada a municípios com os piores indicadores sociais e, em contrapartida, uma maior cobertura de serviços na atenção primária. Embora não seja possível estabelecer uma relação causal, infere-se com base nas perspectivas de plausibilidade e adequação 20 que os resultados do estudo demonstram impactos positivos na saúde bucal do país após a implantação da Política Nacional de Saúde Bucal, como o cumprimento do objetivo de ampliar a oferta dos serviços de atenção secundária em nível nacional sob o princípio da equidade na assistência.

\section{Colaboradores}

M. H. R. Galvão realizou as análises e contribuiu para a redação final do texto. A. G. Roncalli contribuiu na análise dos dados e na redação final do manuscrito.

\section{Informações adicionais}

ORCID: Maria Helena Rodrigues Galvão (00000003-1344-2863); Angelo Giuseppe Roncalli (00000001-5311-697X).

\section{Referências}

1. Ministério da Saúde. Diretrizes da política nacional de saúde bucal. Brasília: Ministério da Saúde; 2004.

2. Departamento de Atenção Básica, Secretaria de Atenção à Saúde, Ministério da Saúde. A saúde bucal no Sistema Único de Saúde. Brasília: Ministério da Saúde; 2018.

3. Ministério da Saúde. Portaria de Consolidação no 5, de 28 de setembro de 2017. Consolidação das normas sobre as ações e os serviços de saúde do Sistema Único de Saúde. Diário Oficial da União 2017; 3 out.

4. Silva HEC, Gottems LBD. Interface entre a Atenção Primária e a Secundária em odontologia no Sistema Único de Saúde: uma revisão sistemática integrativa. Ciênc Saúde Colet 2017; 22:2645-57.

5. Machado FCA, Silva JV, Ferreira MAF. Fatores relacionados ao desempenho de Centros de Especialidades Odontológicas. Ciênc Saúde Colet 2015; 20:1149-63.

6. Goes PSA, Figueiredo N, Neves JC, Silveira FMM, Costa JFR, Pucca JGA, et al. Avaliação da atenção secundária em saúde bucal: uma investigação nos centros de especialidades do Brasil. Cad Saúde Pública 2012; 28 Suppl:S81-9.

7. Herkrath FJ, Herkrath APCQ, Costa LNBS, Gonçalves MJF. Desempenho dos Centros de Especialidades Odontológicas frente ao quadro sociodemográfico dos municípios do Amazonas, Brasil. Saúde Debate 2009; 37:14858.

8. Thomaz EBAF, Sousa GMC, Queiroz RCS, Coimbra LCC. Evaluating productivity targets achievement in Dental Specialty Centers from Maranhão State, Brazil, 2011. Epidemiol Serv Saúde 2016; 25:807-18. 
9. Santana DA, Santos LPS, Carvalho FS, Carvalho CAP. Desempenho dos Centros de Especialidades Odontológicas de uma Região de Saúde da Bahia. Cad Saúde Colet (Rio J.) 2015; 23:261-7.

10. Freitas CHSM, Lemos GA, Pessoa TRRF, Araújo MF, Forte FDS. Atenção em saúde bucal: avaliação dos centros de especialidades odontológicas da Paraíba. Saúde Debate 2016; 40:131-43.

11. Penchansky R, Thomas JW. The concept of access: definition and relationship to consumer satisfaction. Med Care 1981; 19:127-40.

12. Pereira MG. Epidemiologia: teoria e prática. Rio de Janeiro: Editora Guanabara Koogan; 1995.

13. Hair JR, Black WC, Babin BJ, Anderson RE, Tatham RL. Análise multivariada de dados. 6a Ed. Porto Alegre: Bookman; 2009.

14. Programa das Nações Unidas para o Desenvolvimento. Atlas do Desenvolvimento Humano no Brasil. Brasília: Programa das Nações Unidas para o Desenvolvimento; 2013.

15. Corrêa GT, Celeste RK. Associação entre a cobertura de equipes de saúde bucal na saúde da família e o aumento na produção ambulatorial dos municípios brasileiros, 1999 e 2011. Cad Saúde Pública 2015; 31:2588-98.
16. Chaves SCL, Barros SG, Cruz DN, Figueiredo ACL, Moura BLA, Cangussu MCT. Política Nacional de Saúde Bucal: fatores associados à integralidade do cuidado. Rev Saúde Pública 2010; 44:1005-13.

17. Celeste RK, Moura FRR, Santos CP, Tovo MF. Análise da produção ambulatorial em municípios com e sem centros de especialidades odontológicas no Brasil em 2010. Cad Saúde Pública 2014; 30:511-21.

18. Paim JS, Silva LMV. Universalidade, integralidade, equidade e SUS. BIS Bol Inst Saúde 2010; 12:109-14.

19. Lino PA, Werneck MAF, Lucas SD, Abreu MHNG. Análise da atenção secundária em saúde bucal no Estado de Minas Gerais, Brasil. Ciênc Saúde Colet 2014; 19:3879-88.

20. Habicht JP, Victora CG, Vaughan JP. Evaluation designs for adequacy, plausibility and probability of public health programme performance and impact. Int J Epidemiol 1999; 28:10-18. 
Abstract

The study aimed to analyze factors associated with the availability of specialized dental services in Brazilian municipalities. This was an ecological study with a sample of 776 municipalities that participated in the first cycle of the Program to Improve Access and Quality of Dental Specialization Centers (PMAQ-CEO, in Portuguese) survey held in 2014. The study's dependent variables consisted of a coefficient created with variables on the number of professionals and the workweek of dentists in the minimum set of specialties, per 10,000 inhabitants. Exploratory factor analysis was performed to create a score for the municipalities' performance with the availability of specialized dental services. Factors associated with the municipalities' performance were assessed with Pearson's chi-square test, with the following municipal indicators as independent variables, categorized in tertiles: per capita income, Municipal Human Development Index (HDI-M), resident population, total health spending per inhabitant, and Oral Health Teams per 10,000 inhabitants. Higher performance with the availability of specialized oral health services was associated with municipalities having smaller populations (67.3\%; CI: 61.673.0; $p<0.001)$, lower HDI-M (41.9\%; CI: 35.848.0; $p<0.001$ ), lower per capita income (41.2\%; CI: 35.2-47.3; $p<0.001$ ), and higher mean number of oral health teams per 10,000 inhabitants (50.6\%; CI: 46.0-58.4; $p<0.001$ ). The results show positive impacts from the implementation of the National Oral Health Policy in Brazil, meeting the goal of expanding the supply of secondary care services according to the principle of equity in care.

Dental Health Services; Health Care Quality, Access, and Evaluation; Composite Indicators

\section{Resumo}

El objetivo fue analizar nacionalmente los factores asociados con la disponibilidad de servicios odontológicos especializados en municipios brasileños. Se trata de un estudio ecológico, con una muestra de 776 municipios que participaron en el 1er ciclo del Programa para Mejorar el Acceso y la Calidad de los Centros de Especialización Odontológicas (PMAQ-CEO, en Portugués), realizado en 2014. Las variables dependientes del estudio consistieron en un coeficiente creado a partir de las variables de número de profesionales y carga horaria semanal de cirujanos-dentistas, ejerciendo en las especialidades minimas por 10.000 habitantes. Se realizó un análisis factorial exploratorio para la creación de una puntuación que midiera el desempeño de los municipios, respecto a la disponibilidad de servicios odontológicos especializados. Con el fin de evaluar los factores asociados con el desempeño de los municipios, se utilizó el test de chi-quadrado de Pearson, teniendo como variables independientes los siguientes indicadores municipales categorizados en terciles: renta per cápita, Índice de Desarrollo Humano Municipal (IDH-M), población residente, gasto total por habitante en salud y equipos de salud bucal por 10.000 habitantes. El mayor desempeño respecto a la disponibilidad de servicios especializados en salud bucal estuvo asociado a municipios con menor tamaño poblacional (67,3\%; IC: 61,6-73,0; $p<0,001)$, con menor IDH-M (41,9\%; IC: $35,8-48,0 ; p<0,001)$ y con menor renta per cápita (41,2\%; IC: 35,2-47,3; $p<0,001) y$ mayor media de equipos de salud bucal por 10.000 habitantes (50,6\%; IC: 46,0-58,4; $p<0,001$ ). Los resultados demuestran impactos positivos de la implantación de la Politica Nacional de Salud Bucal en el país, como el cumplimiento del objetivo de ampliación de la oferta de los servicios de atención secundaria, basados en el principio de la equidad en la asistencia.

Servicios de Salud Dental; Calidad, Acceso y Evaluación de la Atención de Salud; Indicadores Compuestos
Recebido em 23/Set/2019

Versão final reapresentada em 06/Mai/2020

Aprovado em 09/Jun/2020 